\title{
Role of HSV-2 suppressive therapy for HIV prevention
}

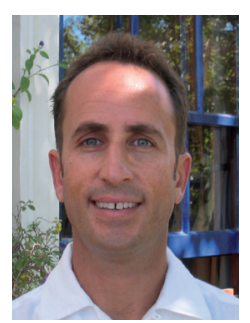

"Until the time when such a vaccine is

developed, combination prevention efforts using

evidence-based strategies, tailored to the

dynamics of individual epidemics, will remain the

cornerstone of HIV prevention."

\section{Steven J Reynolds}

National Institute of Allergy \& Infectious Diseases, National Institutes of Health, MD, USA

= Tel.: +256414323255 = Fax: +256414323252 = sjr@jhmi.edu

Herpes simplex virus type 2 (HSV-2) is one of the most common sexually transmitted infections globally with seroprevalence rates as high as $90 \%$ reported from HIV-infected persons in sub-Saharan Africa [1,2]. The epidemiologic and biologic association between HSV-2 and HIV has been the subject of numerous studies over the past two decades with strong evidence supporting the hypothesis that HSV-2 increases the risk of acquisition among HSV-2-infected, HIVnegative individuals [3-5]. The biological plausibility of this association is explained in part by the portal of entry created during the time of genital ulceration among HSV-2-infected persons and also in part by the influx of HIV target cells that occurs during episodes of HSV-2 reactivation [6,7]. Among individuals coinfected with $\mathrm{HIV}$ and HSV-2, considerable evidence exists to support the hypothesis that HSV-2 also increases the risk of transmission of HIV to uninfected partners because of an increase in HIV plasma viral load, and increased HIV shedding from genital ulcers and the genital tract $[2,8-10]$. This biological synergy between these two viruses has led researchers to consider HSV-2 suppressive treatment as a biomedical prevention strategy to reduce the risk of HIV transmission.

The apparent synergy between HSV-2 and $\mathrm{HIV}$ acquisition prompted researchers to embark on randomized, clinical trials of HSV-2suppressive therapy with the hopes of adding another biomedical HIV prevention strategy to the current prevention options. Two randomized, controlled trials were initiated in 2006 in various sites across sub-Saharan Africa, the USA and Peru to evaluate the impact of HSV-2 suppressive therapy on the risk of HIV acquisition. One trial conducted at a single site in Tanzania enrolled 821 high-risk HIV-negative women who were treated with acyclovir $400 \mathrm{mg}$ twice daily and followed for a minimum of 12 months. Unfortunately, this study showed no difference in HIV incidence between the study arms after 2 years of follow-up (relative risk: 1.08 ; 95\% CI: 0.64-1.83) [11]. A second study conducted among 1814 men who have sex with men in the USA and Peru and 1358 women in South Africa, Zambia and Zimbabwe also failed to show any impact on the risk of HIV acquisition despite high levels of adherence to acyclovir (hazard ratio: 1.16; 95\% CI: 0.83-1.62) [12]. Given the strength of association observed in the epidemiological studies and the supporting biological evidence suggesting an increased risk of HIV acquisition among HSV-2-infected individuals, what could explain the negative findings of these well-conducted clinical trials? Adherence in both studies, as measured by self-report, was high, although the Tanzanian study subsequently investigated drug levels among participants, which suggested the possibility of a lower rate of adherence than measured by self-report, as only $55 \%$ of urine samples from women randomized to the acyclovir arms of the study had detectable acyclovir [13]. The negative results emerging from these two clinical trials have prompted investigators to return to the cellular level to understand the immunobiology of HSV-2 recurrences, which will be discussed later in this article.

More disappointing results emerged recently from a large, multicenter, randomized controlled trial involving $3408 \mathrm{HIV}$-serodiscordant couples where one partner is HIV infected and one is not. In this trial, the HIV/HSV-2 coinfected partner was treated with acyclovir $400 \mathrm{mg}$ twice daily to evaluate the impact on infectiousness to the HIV-uninfected partner. The results of this trial, known as the Partners in Prevention study, were presented at the International AIDS Society meeting last July and revealed no reduction in HIV transmission despite good adherence to acyclovir among treated partners [14]. Although results from this study were disappointing from the prevention standpoint, interesting data were presented on

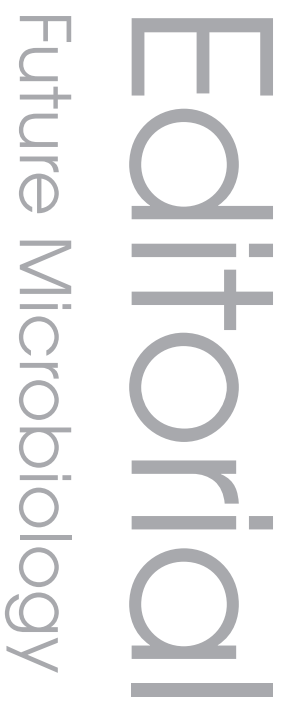

\footnotetext{
"The negative results emerging from these two clinical trials have prompted investigators to return to the cellular level to understand the immunobiology of HSV-2 recurrences..."
}

\section{fulure medicine $_{\text {fisg }}^{\text {ps }}$}




\section{"Clearly, better ways to suppress HSV-2 reactivation and the influx of HIV target cells will need to be developed if this strategy is to have any effect..."}

the role of acyclovir on HIV disease progression among HSV-2/HIV coinfected individuals. The Partners in Prevention study found that acyclovir $400 \mathrm{mg}$ twice daily delayed disease progression (defined as CD4 <250, death due to AIDS or antiretroviral therapy [ART] initiation other than for prevention of mother-to-child transmission) by $17 \%$ [15]. Although the delay in disease progression may be viewed as modest, given the current demand for ART and limited resources in sub-Saharan Africa, this finding may have a role in the pre-ART care of HIV-infected patients with chronic HSV-2. A second study investigating the role of suppressive acyclovir on HIV disease progression in Rakai, Uganda, will be completed in September 2010 and will add additional information on the impact of suppressive acyclovir on HIV disease progression.

How can one interpret the disappointing results of these clinical trials in light of the strong epidemiologic and biologic evidence that HSV-2 increases both acquisition and transmission of HIV? Research carried out at the University of Washington, WA, USA, has shed some light on this by explaining the clinical trial findings by understanding the complexity of what is going on at the cellular level during episodes of HSV-2 reactivation. To understand the immunobiology occurring during periods of HSV-2 reactivation, eight healthy HIV-negative individuals with culture-proven recurrent symptomatic HSV-2 were studied [16]. Punch biopsies were taken during the time of clinically symptomatic ulcerative lesions, at resolution and then at 2, 4 and 8 weeks after healing. Four individuals were then treated with acyclovir $400 \mathrm{mg}$ twice daily at the start of an acute episode and treated for 20 weeks. Biopsies of these individuals were taken again during the acute episode, at resolution and at 2, 4, 8, 12, 16 and 20 weeks. The researchers found evidence of an acute inflammatory response with $\mathrm{CD}^{+}$and $\mathrm{CD} 8^{+}$influx in the epidermis and dermis that persisted for months despite healing of the lesions. Acyclovir treatment was not found to significantly alter this intense inflammatory response even after 20 weeks of therapy. These data help explain why the clinical trials may have failed to interrupt the association between HSV-2 and HIV. Without the ability to diminish the chronic inflammatory milieu resulting from HSV-2, the intervention (acyclovir $400 \mathrm{mg}$ twice daily) may not have been capable of interrupting the biologic interplay between these two viruses.

So what does all this body of evidence leave for the HIV research community looking for ways to stem the current HIV epidemic? Clearly, better ways to suppress HSV-2 reactivation and the influx of HIV target cells will need to be developed if this strategy is to have any effect on reducing the risk of HIV acquisition and transmission. Ideally, an effective HSV-2 vaccine could still have a major impact on reducing HIV transmission given the burden of HSV-2 disease in countries most affected by HIV/AIDS. Until the time when such a vaccine is developed, combination prevention efforts using evidence-based strategies, tailored to the dynamics of individual epidemics, will remain the cornerstone of HIV prevention.

Financial \& competing interests disclosure
The author is employed by and receives research fund-
ing from the Division of Intramural Research, National
Institute of Allergy and Infectious Diseases, NIH. The
author has no other relevant affliations or financial
involvement with any organization or entity with a
financial interest in or financial conflict with the sub-
ject matter or materials discussed in the manuscript
apart from those disclosed.
No writing assistance was utilized in the production
of this manuscript.

\section{Bibliography}

1. Looker KJ, Garnett GP, Schmid GP: An estimate of the global prevalence and incidence of herpes simplex virus type 2 infection. Bull. World Health Organ. 86(10), 805-812 (2008).

2. Mbopi-Keou FX, Gresenguet G, Mayaud P et al.: Interactions between herpes simplex virus type 2 and human immunodeficiency virus type 1 infection in African women: opportunities for intervention. J. Infect. Dis. 182(4), 1090-1096 (2000).
3. Brown JM, Wald A, Hubbard A et al.: Incident and prevalent herpes simplex virus type 2 infection increases risk of HIV acquisition among women in Uganda and Zimbabwe. AIDS 21(12), 1515-1523 (2007).

4. Freeman EE, Weiss HA, Glynn JR, Cross PL, Whitworth JA, Hayes RJ: Herpes simplex virus 2 infection increases HIV acquisition in men and women: systematic review and meta-analysis of longitudinal studies. AIDS 20(1), 73-83 (2006).

5. Reynolds SJ, Risbud AR, Shepherd ME et al.: Recent herpes simplex virus type 2 infection and the risk of human immunodeficiency virus type 1 acquisition in India. J. Infect. Dis. 187(10), 1513-1521 (2003).

6. Koelle DM, Abbo H, Peck A, Ziegweid K, Corey L: Direct recovery of herpes simplex virus (HSV)-specific $\mathrm{T}$ lymphocyte clones from recurrent genital HSV-2 lesions. J. Infect. Dis. 169(5), 956-961 (1994).

7. Zhu J, Koelle DM, Cao J et al.: Virus-specific $\mathrm{CD}^{+} \mathrm{T}$ cells accumulate near sensory nerve endings in genital skin during subclinical HSV-2 reactivation. J. Exp. Med. 204(3), 595-603 (2007). 
8. McClelland RS, Wang CC, Overbaugh J et al: : Association between cervical shedding of herpes simplex virus and HIV-1. AIDS 16(18), 2425-2430 (2002).

9. Mole L, Ripich S, Margolis D, Holodniy M: The impact of active herpes simplex virus infection on human immunodeficiency virus load. J. Infect. Dis. 176(3), 766-770 (1997).

10. Schacker T, Ryncarz AJ, Goddard J, Diem K, Shaughnessy M, Corey L: Frequent recovery of HIV-1 from genital herpes simplex virus lesions in HIV-1-infected men. JAMA 280(1), 61-66 (1998).

11. Watson-Jones D, Weiss HA, Rusizoka M et al:: Effect of herpes simplex suppression on incidence of HIV among women in Tanzania. N. Engl. J. Med. 358(15), 1560-1571 (2008).

12. Celum C, Wald A, Hughes J et al.: Effect of aciclovir on HIV-1 acquisition in herpes simplex virus 2 seropositive women and men who have sex with men: a randomised, double-blind, placebo-controlled trial. Lancet 371(9630), 2109-2119 (2008).
13. Watson-Jones D, Baisley K, Rusizoka M et al.: Measurement and predictors of adherence in a trial of HSV suppressive therapy in Tanzania. Contemp. Clin. Trials (2009) (Epub ahead of print).

14. Celum C, Wald A, Lingappa J et al.: Twice-daily acyclovir to reduce HIV-1 transmission from HIV-1 / HSV-2 co-infected persons within HIV-1 serodiscordant couples: a randomized, double-blind, placebocontrolled trial. Presented at: 5th International AIDS Society Conference on HIV Pathogenesis, Treatment and Prevention. Cape Town, South Africa, 19-22 July 2009

(Abstract WELBC101).

15. Lingappa JR, Baeten JM, Wald A et al:: Daily acyclovir delays HIV-1 disease progression among HIV-1/HSV-2 dually-infected persons: a randomized trial. Presented at: 5 th International AIDS Society Conference on HIV Pathogenesis, Treatment and Prevention. Cape Town, South Africa, 19-22 July 2009 (Abstract WELBC102).
16. Zhu J, Hladik F, Woodward A et al:: Persistence of HIV-1 receptor-positive cells after HSV-2 reactivation is a potential mechanism for increased HIV-1 acquisition. Nat. Med. 15(8), 886-892 (2009).

\section{Affiliations}

- Steven J Reynolds

Laboratory of Immunoregulation,

National Institute of Allergy \& Infectious Diseases, National Institutes of Health, MD, USA

and

Johns Hopkins University School of Medicine, MD, USA

Tel.: +256414323255

Fax: +256414323252

sjr@jhmi.edu 